\title{
BREAST CANCER IN TWIN PREGNANCY
}

Camila Farabotti Matheus¹, Tayana Moreira de Faria¹, Andre Mattar ${ }^{1}$, Jorge Yoshinori Shida', Luiz Henrique Gebrim

${ }^{1}$ Hospital Perola Bygnton - São Paulo (SP), Brazil.

Introduction: Breast cancer is the most common neoplasm among pregnant women, and occurs in up to $0.04 \%$ of pregnancies. There are only few short and long term results about the intrauterine exposure to antineoplastic agents, and treatment should follow the same guidelines as the one for non-pregnant women. Surgery is possible during the entire pregnancy. Radiotherapy is not indicated in pregnancy, but it is possible for selected patients. Chemotherapy is usually safe during the second and third trimesters, with interruption of the treatment three weeks before the probable date of birth; however, hormone therapy and anti-HER 2 agents are contraindicated during pregnancy, and can be postponed until after birth. Objective: To report the case of a patient with breast cancer in the first trimester of a monochorionic diamniotic twin pregnancy, 13 years after thoracic radiotherapy due to Hodgkin's lymphoma. Methods: Medical chart review, interview with the patient, photographic record and literature review. Results: Thirty-four year old Japanese descendant woman, born in São Paulo, nutritionist, married, primiparous. In the eighth week of spontaneous twin pregnancy, she complained of a palpable, fast-growth nodule in the left breast superomedial quadrant. She took combined hormonal contraceptives from the ages of 17 to 33 years. Negative family history for neoplasms. Physical examination showed a nodule in the left breast superomedial quadrant measuring 9.0x7.5 cm - T3N0 (initial clinical staging IIB). Propedeutics: Obstetric ultrasound with topic monochorionic diamniotic twin pregnancy, compatible with 13 weeks and 5 days; breast US showed a complex irregular nodule in the left breast at $9 \mathrm{~h}$, measuring $54 \times 40 \times 40 \mathrm{~mm}$ : Birads 4 . The ultrasound-guided percutaneous biopsy showed invasive carcinoma without expression of hormone receptors, negative HER2, 95\% KI67 (triple negative). Negative staging for metastasis. She was referred to clinical oncology: received five sessions of Carboplatin (AUC 2) and Paclitaxel $\left(80 \mathrm{mg} / \mathrm{m}^{2}\right)$. Despite the treatment, the tumor progressed to $12 \mathrm{x} 10.5 \mathrm{~cm}$, with large areas and negative axilla. The choice was for mastectomy with axillary lymphadenectomy. Anatomopathological: absence of residual neoplastic cells and neoplasm in axillary nodes (0/8): pathological complete response. A Cesarean section was performed after 36 weeks of pregnancy, and two female infants were born, weighing 2030g and 2455g, Apgar 8/9 and 9/9, respectively. Conclusion: Breast cancer diagnosis and prognosis during pregnancy are not well established. Considering that, this study collaborates with others that were conducted to better understand this pathology in twin pregnancies. 